\title{
Article \\ Silicon Nanowire Field-Effect Transistor as Label-Free Detection of Hepatitis B Virus Proteins with Opposite Net Charges
}

\author{
Suh Kuan Yong ${ }^{1}$, Shang-Kai Shen ${ }^{2}$, Chia-Wei Chiang ${ }^{2}$, Ying-Ya Weng ${ }^{2}$, Ming-Pei Lu ${ }^{3, *}$ \\ and Yuh-Shyong Yang $1, * \mathbb{C}$
}

1 Department of Biological Science and Technology, National Yang Ming Chiao Tung University, Hsinchu 300, Taiwan; wendyyongsk.bt05g@nctu.edu.tw

2 Institute of Biomedical Engineering, National Yang Ming Chiao Tung University, Hsinchu 300, Taiwan; fr509.cm05g@nctu.edu.tw (S.-K.S.); chiangwill0813@gmail.com (C.-W.C.); lynneweng8571@gmail.com (Y.-Y.W.)

3 Taiwan Semiconductor Research Institute, National Applied Research Laboratories, Hsinchu 300, Taiwan

* Correspondence: mplu@narlabs.org.tw (M.-P.L.); ysyang@mail.nctu.edu.tw (Y.-S.Y.)

Citation: Yong, S.K.; Shen, S.-K.; Chiang, C.-W.; Weng, Y.-Y.; Lu, M.-P.; Yang, Y.-S. Silicon Nanowire Field-Effect Transistor as Label-Free Detection of Hepatitis B Virus Proteins with Opposite Net Charges. Biosensors 2021, 11, 442. https:// doi.org/10.3390/bios11110442

Received: 17 September 2021 Accepted: 4 November 2021 Published: 10 November 2021

Publisher's Note: MDPI stays neutral with regard to jurisdictional claims in published maps and institutional affiliations.

Copyright: (c) 2021 by the authors. Licensee MDPI, Basel, Switzerland. This article is an open access article distributed under the terms and conditions of the Creative Commons Attribution (CC BY) license (https:// creativecommons.org/licenses/by/ $4.0 /)$

\begin{abstract}
The prevalence of hepatitis B virus (HBV) is a global healthcare threat, particularly chronic hepatitis B (CHB) that might lead to hepatocellular carcinoma (HCC) should not be neglected. Although many types of HBV diagnosis detection methods are available, some technical challenges, such as the high cost or lack of practical feasibility, need to be overcome. In this study, the polycrystalline silicon nanowire field-effect transistors (pSiNWFETs) were fabricated through commercial process technology and then chemically functionalized for sensing hepatitis B virus surface antigen (HBsAg) and hepatitis B virus X protein ( $\mathrm{HBx}$ ) at the femto-molar level. These two proteins have been suggested to be related to the HCC development, while the former is also the hallmark for HBV diagnosis, and the latter is an RNA-binding protein. Interestingly, these two proteins carried opposite net charges, which could serve as complementary candidates for evaluating the chargebased sensing mechanism in the pSiNWFET. The measurements on the threshold voltage shifts of pSiNWFETs showed a consistent correspondence to the polarity of the charges on the proteins studied. We believe that this report can pave the way towards developing an approachable tool for biomedical applications.
\end{abstract}

Keywords: hepatitis B virus; hepatitis B virus surface antigen (HBsAg); hepatitis B virus X protein (HBx); chronic hepatitis B (CHB); hepatocellular carcinoma (HCC); biomarker; protein-protein interaction; biosensor; silicon field-effect transistor (SiNWFET); polycrystalline silicon field-effect transistor (pSiNWFET)

\section{Introduction}

Hepatitis B virus (HBV) belongs to the Hepadnaviridae family. The $42 \mathrm{~nm}$ virion consists of a lipoprotein outer envelope and a nucleocapsid core antigen containing circular, partly double-stranded viral DNA [1]. The viral DNA size is around $3.2 \mathrm{~kb}$ with four known genes (e.g., S, C, P, and X) [1,2]. These four genes are overlapping and known as open reading frames (ORF), which $S$ gene, $C$ gene, $P$ gene, and $X$ gene encode for surface protein, core protein and e antigen, polymerase and reverse transcriptase, and $x$ protein, respectively [1]. The infection of HBV causes acute and chronic hepatitis B (CHB) in humans. The number of people with chronic hepatitis $\mathrm{B}(\mathrm{CHB})$ condition was estimated at 257 million in 2015 [3]. It is known that CHB patients have a higher risk of developing liver fibrosis, cirrhosis, and hepatocellular carcinoma (HCC) [3]. With the adoption of the 2030 Agenda for Sustainable Development, World Health Organization (WHO) has set a goal to eliminate viral hepatitis by 2030 to reduce chronic hepatitis incidence and annual deaths to 0.9 million and less than 0.5 million, respectively [4]. 
Hepatitis B virus surface antigen (HBsAg) was discovered in 1965, and it became the hallmark of early diagnosis marker for HBV infection diagnosis [5]. The HBsAg is a glycosylated envelope protein of HBV virion, which composes three parts (e.g., small, medium, and large proteins) [5]. The development of immunoassays that achieved a lower detection limit and the ability to quantify HBsAg commercial kits available has widened interest study on HBsAg [6]. There are two main HBsAg quantification methods, which include Architect HBsAg QT (Abbott Diagnostics) and Elecsys HBsAg II (Roche Diagnostics) [5], and the former is most widely being used. The quantification of HBsAg allows the scientific community to analyze the correlation of intrahepatic HBV DNA and covalently closed circular DNA (cccDNA level) [6]. These findings suggested that HBsAg could be the surrogate marker in monitoring CHB patients for drugs treatment response, viral control status, and prognosis of hepatitis [7]. Besides that, hepatitis $B$ virus $\mathrm{X}$ protein $(\mathrm{HBx})$ also has been proven to play a crucial role in hepatocarcinogenesis. Adequate evidence suggested that the HBx acts as a pleiotropic transactivator in many HCC development cellular pathways, including interaction with damage-specific DNA-binding protein 1 [8], centrosomal P4.1-associated protein [9], and nucleic acid metabolism [10]. A recent study also reported that the HBx might bind to the promoter region of DLEU2 long non-coding RNAs, which are frequently dysregulated in HBV-related HCC [11]. These findings showed that $\mathrm{HBx}$ could be a potential biomarker for monitoring the pathogenesis of HCC from CHB patients.

HBV vaccination was applied a few decades ago, and the vaccination program has reduced HBV prevalence in many countries. However, in developing countries, HBV prevalence remains high due to the poor prevention, management, and control of the virus infection spreading [12]. The poor virus infection control could be due to many factors such as ignorance, high cost of the vaccination program, lack of healthcare facilities, lack of trained diagnostic personnel, and the treatment protocol extrapolated from developed countries might not be appropriate for developing countries [13]. According to the WHO report, less than $5 \%$ of $\mathrm{CHB}$ patients know their status, and expensive lifelong diagnostic is unaffordable for most countries [4]. A constant checkup is necessary to monitor CHB and prevent the virus from outspreading during the incubation period. Besides that, the CHB patients might be asymptomatic and cause the virus to spread silently [14]. Thus, it is necessary to develop a simple, low-cost, and ultra-sensitive HBV detection method for sensing key biomarkers, such as HBsAg and $\mathrm{HBx}$, to provide a sustainable diagnosis of $\mathrm{HBV}$ infection and early diagnosis of CHB patients.

To date, a broad diversity of field-effect transistor (FET) sensors have been invented and studied for their potential application in the biomedical field [15]. Among these FET sensors, the silicon nanowire field-effect transistor (SiNWFET) sensor shows a label-free sensing principle, and field-effect interaction has attracted significant attention [16]. In addition, the well-developed silicon industry is beneficial to the study of SiNWFET. Many studies have demonstrated the potential of SiNWFET for biosensing applications, including detection of nucleic acids [17], antibodies and antigens [18], and protein-protein interactions [19]. These findings revealed the benefits of SiNWFET, including ultrahigh-sensitivity, real-time, and label-free detection, making it a great potential candidate in biosensor development $[20,21]$. Generally, an electronic biosensor composed of a bio-receptor and electronic transducer allows for converting the biological information into an electronic signal [16]. Thereby, the direct conversion of a biological reaction to electronic signals has widened the biological applications of the biosensor. Furthermore, the integration of biosensors with an innovative analytic framework could make it act as a good biosensor candidate for point-of-care testing (POCT) and the internet of things (IoT) [22-24].

Due to the technical difficulties in the bottom-up fabrication process of SiNWFET, a simple and cost-effective polycrystalline silicon sidewall spacer etching technique has been proposed [25-28]. This process technique is compatible with the complementary metaloxide-semiconductor (CMOS) process, which is commonly used in the current commercial semiconductor process. Accordingly, the polycrystalline silicon nanowire field-effect 
transistors (pSiNWFETs) in this report were fabricated by the commercial process foundry through the sidewall spacer etching technique. Furthermore, the chemical functionalization techniques of the pSiNWFET surface were performed for the immobilization of the relevant antibodies for sensing the HBV-related biomarkers such as HBsAg and HBx proteins. The HBsAg is the hallmark of HBV infection diagnosis and has been discussed in other papers $[29,30]$. However, HBx protein detection is a new topic that has not been addressed in other articles. Notably, these two proteins possess opposite polarities of the charges to each other, revealing the negative and positive polarities for HBsAg and HBx proteins, respectively. The difference between the threshold voltage of pSiNWFETs before and after the antigen incubation revealed a consistent correspondence to the polarity of the charges on the proteins. By varying the concentrations of the antigens, the equilibrium dissociation constant $\left(K_{D}\right)$ can be extracted, showing the value at the femto-molar level for HBsAg and $\mathrm{HBx}$ cases. Our experimental demonstration of using pSiNWFETs as new biosensors to detect the HBsAg and HBx proteins at the femto-molar level provides an opportunity to evaluate and understand hepatitis infection and future biosensor development for the realm of POCT.

\section{Materials and Methods}

\subsection{Materials}

Acetone $(99.9 \%)$ and ethanol $(99.5 \%$ and $99.8 \%)$ were obtained from ECHO Chemical Co., Ltd. (Miaoli, Taiwan). Glutaraldehyde (GA), 3-aminopropyltrithoxysilane (APTES), sodium cyanoborohydride $\left(\mathrm{NaBH}_{3} \mathrm{CN}\right)$, Bis-tris propane, and anti-mouse IgG (whole molecule)-gold antibody (Lot. Number G7652) were obtained from Sigma (St. Louis, MO, USA). Hepatitis B surface antibody (HBsAb, Lot. Number GTX36859), hepatitis B surface antigen (HBsAg, Lot. Number GTX57164), hepatitis B virus X protein antibody (anti-HBx, Lot. Number GTX22741), and hepatitis B virus X protein (HBx, Lot. Number GTX17526-pro) were obtained from Genetex Inc. (Irvine, CA, USA). EKC830 was obtained from DuPont Electronics Technologies, USA.

\subsection{Device Fabrication}

The n-type pSiNWFET were fabricated using a commercial process technology provided by Episil Holding Inc. Taiwan. The pSiNWFET structure comprised two poly-silicon nanowires with $94 \mathrm{~nm}$ in width and $2.43 \mu \mathrm{M}$ in length, which served as conducting channels. The fabrication procedure was performed using the sidewall spacer technique, which has been developed previously [25-27].

\subsection{Device Cleaning, Surface Modification, and Antibody Immobilization}

The pSiNWFET was treated with organic solvents, including EKC830 and $99.5 \%$ ethanol, to remove the surface of unwanted chemical compounds and the photoresist layer. The EKC 830 was heated to $95^{\circ} \mathrm{C}$ before pSiNWFET soaked into for $10 \mathrm{~min}$ and washed with $99.5 \%$ ethanol.

Subsequently, the chemical surface modification for self-assembly of antibodies on pSiNWFET was performed. First, the pSiNWFET was cleaned with plasma cleaner (Harrick Plasma PDC-32G) for 5 min before being immersed into 2\% APTES diluted in 99.8\% ethanol solution to form a self-assembled monolayer which covalently links between surface silanol groups $(\mathrm{SiOH})$ and terminal with amines groups $\left(\mathrm{NH}_{2}\right)$. Subsequently, the pSiNWFET was cleaned with $99.5 \%$ ethanol and heated on a hot plate at $120^{\circ} \mathrm{C}$ to remove surplus ethanol. Second, the pSiNWFET was soaked into $2.5 \%$ GA mixed in $10 \mathrm{mM}$ Bis-tris propane solution for $30 \mathrm{~min}$, forming a connection of amines group from APTES and a terminal of aldehyde group.

Antibody immobilization was performed by adding $1 \mu \mathrm{g} / \mathrm{mL}$ of $\mathrm{HBsAb}$ or $10 \mu \mathrm{g} / \mathrm{mL}$ of anti-HBx that functioned as a probe onto the device surface and incubated for $16 \mathrm{~h}$ at $4{ }^{\circ} \mathrm{C}$. The amino acid of the antibody will bind to the aldehyde group of GA. The nonspecific binding sides and active amine groups were blocked by $4 \mathrm{mM} \mathrm{NaBH}{ }_{3} \mathrm{CN}$ solution 
containing $10 \mathrm{mM}$ Tris- $\mathrm{HCl}$ buffer (Scheme 1). The pSiNWFET was dried with nitrogen gas and kept in a vacuum bag at $4{ }^{\circ} \mathrm{C}$ for further experiments.

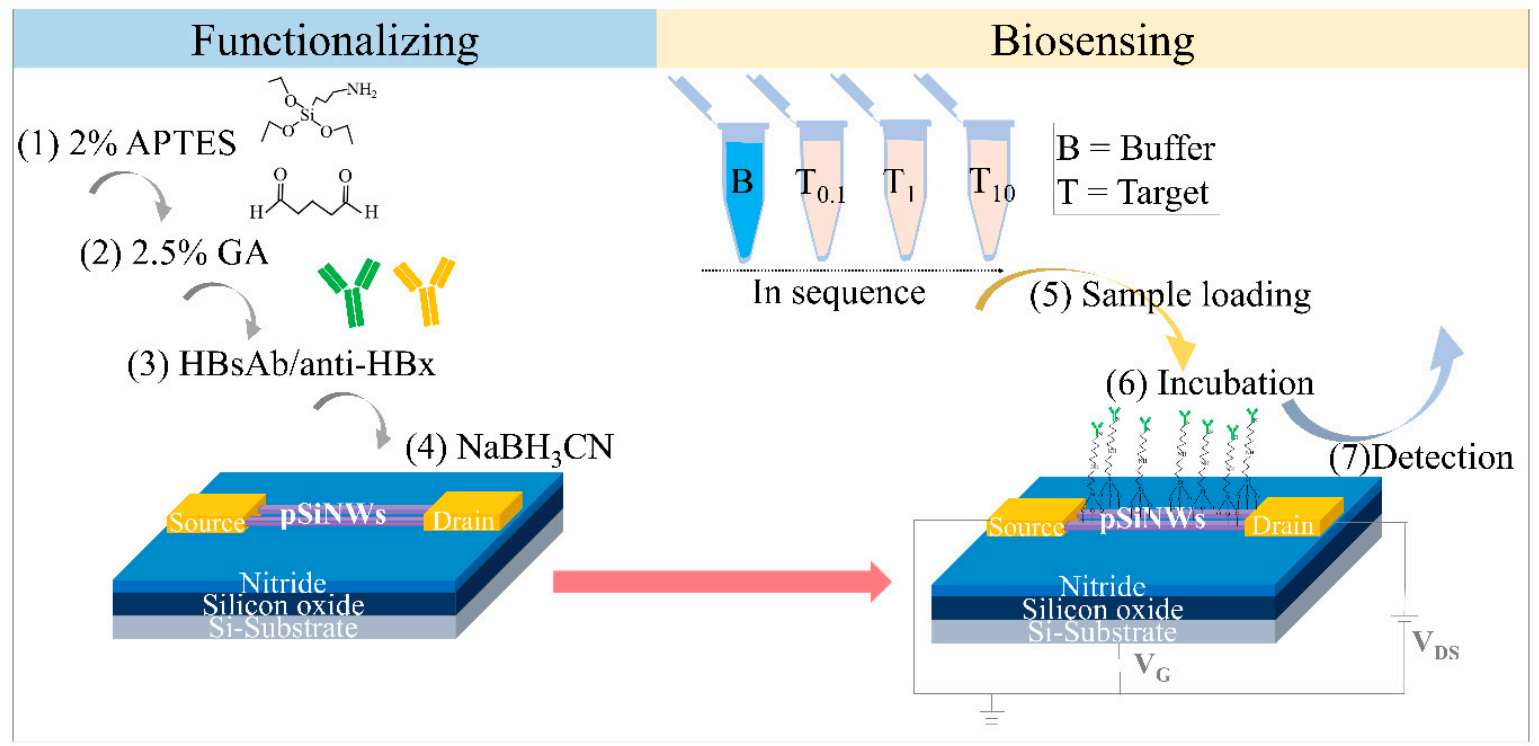

Scheme 1. Schematic illustration of a pSiNWFET (not in scale) functionalizing procedure and the flow of sample detection. (1-4) The step of a device surface modification and HBsAb or anti-HBx immobilization. (5-7) The HBsAg or HBx biosensing step using a functionalized pSiNWFET, the HBsAb-HBsAg, and anti-HBx-HBx interaction will be detected via the electrical property response of the biosensor.

\subsection{Surface Modification and Probe Immobilization Verification}

X-ray photoelectron spectroscopy (XPS) surface analysis was performed using a PHI Quantera II with an X-ray spot size of $200 \mathrm{~nm}$. This experiment was performed to verify the surface modification step and success of the probe immobilization. The silicon wafer before and after surface modification, as described in Section 2.3 were analyzed its element of carbon $(\mathrm{C})$, nitrogen $(\mathrm{N})$ and oxygen $(\mathrm{O})$ (Figure $\mathrm{S} 1 \mathrm{in}$ Supplementary Materials).

A scanning electron microscope (SEM) was used to verify surface modification and probe immobilization on the device. The anti-mouse-gold antibody was used to interact with the immobilized HBsAb. The anti-mouse-gold antibody is an anti-mouse IgG antibody conjugated nano-gold particle with a size range of 10 to $12 \mathrm{~nm}$. The nano-gold particles will be observed using SEM. The anti-mouse-gold antibody was prepared with $0.1 \%$ phosphatebuffered saline (1:100) and loaded onto the pSiNWFET before and after pSiNWFET surface modification mentioned in Section 2.3. The anti-mouse-gold antibody was incubated for $2 \mathrm{~h}$ at room temperature. Subsequently, the anti-mouse-gold antibody from each pSiNWFET was washed with deionized water and dried with nitrogen gas. The pSiNWFET was coated with a layer of platinum with a power range of 10 to $30 \mathrm{~mA}$ for a period range of 10 to $50 \mathrm{~s}$. The pSiNWFET and nano-gold particles were observed under the SEM setting of IST, $10.0 \mathrm{kv}, 8.5-8.7 \mathrm{~mm}, 50.0 \mathrm{k}$ magnification, and SE(U).

\subsection{Electrical Property of $p$ SiNWFET Measurement}

The electrical characterization measurement of pSiNWFET was performed by employing a commercial characterization analyzer (Keithley 2636a). During the electrical measurement of the $\mathrm{I}_{\mathrm{D}}-\mathrm{V}_{\mathrm{G}}$ curve, the drain voltage was set at a constant value of $0.5 \mathrm{~V}$ $\left(\mathrm{V}_{\mathrm{D}}=0.5 \mathrm{~V}\right)$, and the gate voltage was swept from -1 to $2 \mathrm{~V}$. To screen the devices with more reliability, we intentionally measured the $\mathrm{I}_{\mathrm{D}}-\mathrm{V}_{\mathrm{G}}$ curve of each device for three times to check whether there was any evidence of variation over time. The baseline of the $I_{D}-V_{G}$ curve was measured under aqueous conditions (Figure S2A). 


\subsection{Extraction of Threshold Voltage Shift in pSiNWFET}

As aforementioned, the baselines of the electrical properties of pSiNWFETs were measured before loading the analyte samples onto the pSiNWFET surface. Each device was incubated with analyte solution (e.g., HBsAg or HBx) for $30 \mathrm{~min}$. The analyte was prepared in the concentration of $100 \mathrm{fg} / \mathrm{mL}$ to $10 \mathrm{pg} / \mathrm{mL}$ in $10 \mathrm{mM}$ of Bis-tris propane ( $\mathrm{pH} 7)$. Then, it was followed by performing a washing process of the device surface with $10 \mathrm{mM}$ of Bis-tris propane buffer. Later, the electrical property measurement was performed, as described in Scheme 1. The difference between pSiNWFET threshold voltage before and after analyte incubation was extracted as the sensing signal. The threshold voltage $\left(V_{\text {th }}\right)$ was determined with the intercept of the linear approximation of the $I_{D}-V_{G}$ curve at the maximum slope point, as shown in Figure S3 [31]. Subsequently, the threshold voltage changing $\left(\Delta \mathrm{V}_{\text {th }}\right)$ was obtained which the value of analyte $\mathrm{V}_{\text {th }}$ subtracts the value of baseline $V_{\text {th }}$.

\subsection{Extraction of the Equilibrium Dissociation Constant $\left(K_{D}\right)$ in $p$ SiNWFET}

The data collected from Section 2.6 was further evaluated for obtaining $K_{D}$ of $\mathrm{HBsAg}$ and HBx in pSiNWFET using the following equation [32]:

$$
\frac{\Delta I_{d s}}{g_{m}}=\Delta V_{T}=\frac{q_{A}}{C_{0}}[B]_{\max } \times \frac{[A]}{[A]+K_{D}}
$$

where $q_{A}$ is the absorbed analytes electric charge, $C_{0}$ is the analyte/channel capacitive coupling, $[A]$ is the analyte concentration in bulk solution, and $[B]_{\max }$ is the maximum surface density of functional binding sites on silicon nanowire [32].

\subsection{Protein Zeta Potential Measurement}

The zeta potential was performed using Zeta-potential \& Particle size Analyzer ELSZ2000 series (Qtsuka Electronics Co., Ltd.) and Zetasizer Nano ZS90 instrument (Malvern Instruments Ltd., United Kingdom). The proteins HBsAg and HBx were individually prepared in $10 \mathrm{mM}$ Bis-tris propane, $\mathrm{pH} 7$, at the concentration of $6.25 \mu \mathrm{g} / \mathrm{mL}$. The refractive index, dielectric constant, and viscosity of the instrument were set at 1.5650, 78.3, and $0.8878 \mathrm{cP}$ at $25^{\circ} \mathrm{C}$, respectively. The zeta potential of the protein was measured thrice, and the average value was calculated.

\section{Results and Discussion}

\subsection{Device Structure}

Figure 1A shows the schematic of the structure of the pSiNWFET (not in scale). The structure includes a silicon substrate, a stacked silicon nitride/silicon oxide layer, two highly doped Si areas, and two poly-Silicon nanowires (pSiNWs) acts as the back-gate electrode, the gate dielectric, source/drain area, and the conducting channels, respectively. The top-view SEM image of the device is demonstrated in Figure 1B, revealing that two nanowires (NWs) are located on the surface of the nitride layer. The length and width of the NWs are measured to be $2.43 \mu \mathrm{M}$ and $94 \mathrm{~nm}$, respectively. Besides that, the source and drain are covered with the passivation layer to prevent direct contact with the aqueous and cause a short circuit (Figure S4).

There are two kinds of technical approaches, top-down and bottom-up, for the semiconductor process fabrication of SiNWFET. The top-down approach involves using advanced lithography and reactive ion etching techniques to fabricate the NW structures on the surface of the wafer. In contrast, the bottom-up approach is based on the assembling technique of the NWs grown by the vapor-liquid-solid method to place the NWs on the wafer surface [33]. Although the top-down fabrication approach is amenable to mass production, reliable and reproducible, and has no integration issues. However, it is costly, limited in NW dimension and limited choice of NW material, and time-consuming [34]. The previous studies showed a simple and cost-effective top-down fabrication using the sidewall spacer etching technique [25-28] that was transferred to a commercial foundry 
via an industry-academia collaboration cooperation project. The pSiNWFET devices used in this study supplied by the commercial semiconductor company showed that the fabrication technique of pSiNWFET was successfully transferred from an academic laboratory (National Applied Research Laboratories, Taiwan Semiconductor Research Institute) to a commercial foundry. Figure S2B shows the electrical characteristics measured from nine devices, revealing the excellent and reliable features. It indicates the mass production of the pSiNWFET by the commercial foundry is highly feasible.

(A)

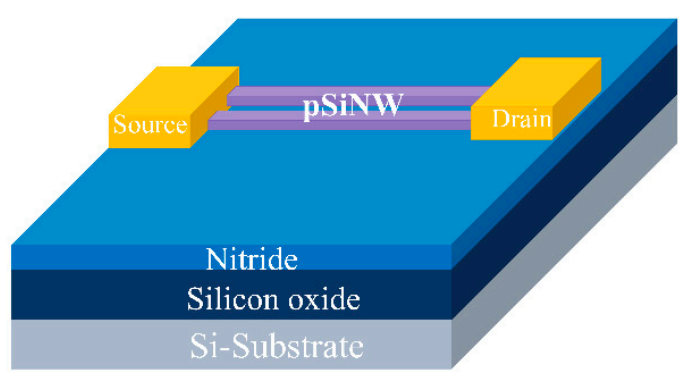

(B)

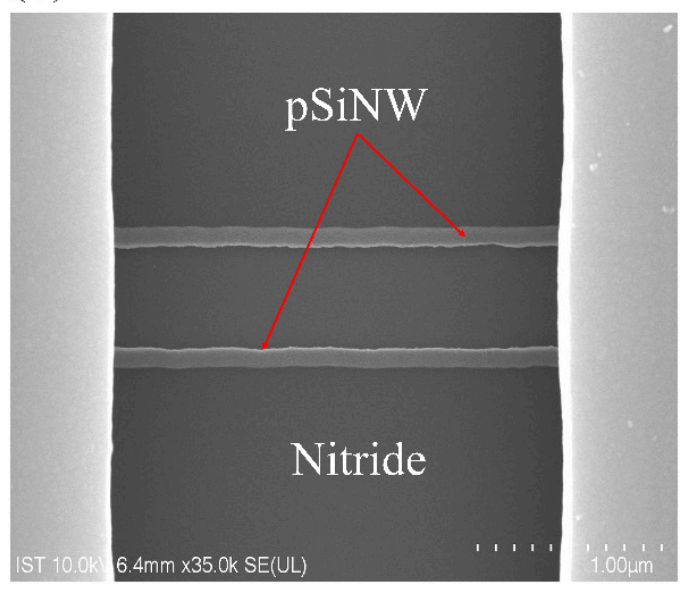

Figure 1. Schematic diagram and SEM images of the pSiNWs structure. (A) The schematic diagram of a pSiNWFET structure includes the layer of silicon oxide, nitride, source/drain, and two pSiNWs (not in scale). (B) The SEM image of a device from top-view with $35 \mathrm{k}$ of magnification. The two pSiNWs lengths and widths were $2.43 \mu \mathrm{M}$ and $94 \mathrm{~nm}$, respectively.

\subsection{Surface Modification and Probe Immobilization Verification}

Surface modification and immobilization of antibodies on the devices is the first critical step that needs to be achieved for developing a biosensor. In this study, the surface modification and self-assembly antibodies on the device with amine and aldehyde linkers were adapted from the previous study [35]. The verification was analyzed using XPS (Figure 2), which XPS is a powerful tool used to analyze surface chemistry [36]. The four surface modifications on silicon wafer include nude, APTES modified, APTES + glutaraldehyde modified (APTES + GA) APTES + glutaraldehyde + HBsAb modified (APTES + GA + HBsAb) were analyzed, as shown in Figure 2. The XPS C1s spectrum showed a peak at $283.5 \mathrm{eV}$ for the nude surface (Figure 2A). Following the surface modification steps, an increased intensity major peak has been observed at the binding energy range of $283.5 \mathrm{eV}$ to $284.5 \mathrm{eV}$, which may be attributed to C-C/C-H. The increased intensity of the major peak suggests that more carbon elements were found on the surface, which supported the longer linkers immobilized along with the immobilization steps. Other than the nude silicon wafer, minor peaks were observed at the binding energy range of $287.5 \mathrm{eV}$ to $288.5 \mathrm{eV}$ after APTES immobilization, which may be attributed to $\mathrm{C}-\mathrm{N} / \mathrm{C}-\mathrm{O} / \mathrm{C}=\mathrm{O}[37,38]$. Figure 2B shows the XPS spectrum of nitrogen element of four surface modifications as aforementioned. The peaks observed at $399 \mathrm{eV}$ show increased intensities after being modified with APTES + GA + HBsAb. This result suggested more nitrogen elements were found on the surface and supported longer linkers immobilized on the surface, particularly APTES and antibodies containing nitrogen elements. Similarly, the oxygen element of four surface modifications was analyzed at the binding energy at $532 \mathrm{eV}$ (Figure S1). 

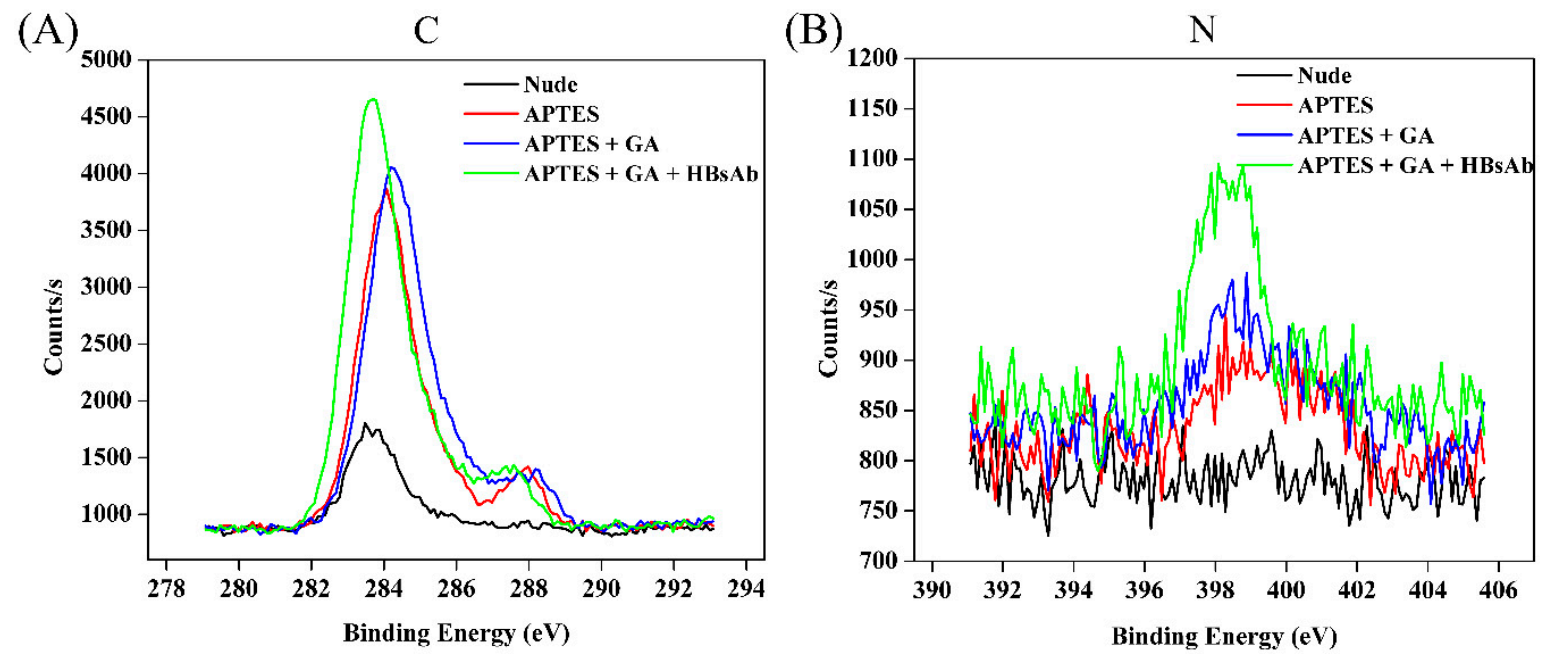

Figure 2. XPS carbon and nitrogen spectrum of silicon wafer (Si-wafer) surface modification, and HBsAb immobilization procedure. Nude, APTES, APTES + GA, and APTES + GA + HBsAb indicate the Si-wafer without surface modification, after being treated with APTES, after being treated with APTES and glutaraldehyde, and after being treated with APTES, glutaraldehyde, and HBsAb, respectively. (A) Carbon XPS spectra and (B) Nitrogen XPS spectra at each immobilization step.

Furthermore, we used the SEM tool to verify the validity of the surface modification on devices. The anti-mouse IgG-gold antibody (AuNPs) was used to confirm the success of probe immobilization on pSiNWFET by binding on the immobilized HBsAb, as shown in Figure S5. Figure S5 showed the SEM analysis of the nude device (Figure S5A) and APTES + glutaraldehyde + HBsAb modified device (Figure S5B). The AuNPs specifically bind to the HBsAb but cannot be linked to the nude pSiNWFET. Figure S5D showed increased quantities of nano-gold particles that have been observed on functionalized pSiNWFET. These results showed that only the devices with immobilized antibodies observed expected nano-gold particles and indicates that the probes were successfully immobilized onto the pSiNWFET.

\subsection{Electrical Properties Measurement of Surface Modification and Probe Immobilization}

The procedure of probe immobilization was confirmed again by measuring the electrical properties changes, which has been reported in a previous study [35]. As shown in Figure 3, the electrical properties of pSiNWFET were measured following surface modification steps. Figure 3 showed nude device electrical property (G1, black line) and served as the baseline of the device. Then, APTES modified device was measured (G2, red line). The increased $I_{D}$ and decrease in threshold voltage were observed when there is excess positive charge available on the surface for an n-type pSiNWFET. The excess positive charge was contributed by the amine group of APTES $(\mathrm{pKa}=4.0)$ at $\mathrm{pH}$ 7. Subsequently, the APTES + glutaraldehyde modified pSiNWFET showed a decrease in $I_{D}$ (G3, blue line), which was caused by the imide bonds formation of the glutaraldehyde, where the positive charge of the amine group of APTES was converted to neutral by the imide bonds. Lastly, the immobilized $\mathrm{HBsAb}\left(\mathrm{G} 4\right.$, green line) showed a decrease in $\mathrm{I}_{\mathrm{D}}$ indicated excess negative charges applied onto the device. The inset figure represents the change in threshold voltage following each surface modification step. This result is consistent with our previous studies $[19,35]$, which determine the surface modification process by measuring the electrical properties of pSiNWFET. 


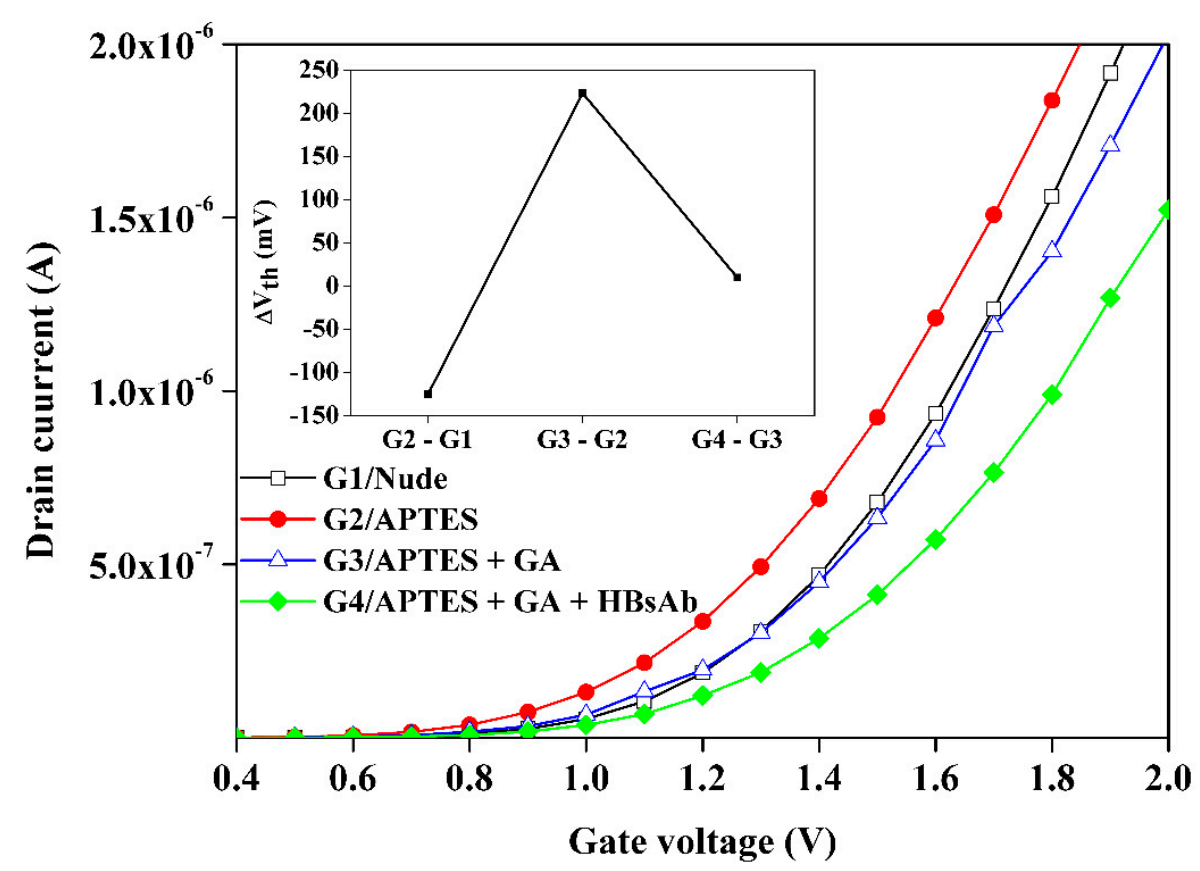

Figure 3. The electrical properties of pSiNWFET following each step of surface modification and HBsAb immobilization. $\mathrm{I}_{\mathrm{D}}-\mathrm{V}_{\mathrm{G}}$ linear graph was analyzed from nude (G1), treated with APTES (G2), APTES + GA (G3), and APTES + GA + HBsAb (G4). The inset figure showed the changes of threshold voltage following each step of surface modification.

\subsection{Biosensing of Various Concentrations of HBsAg and $H B x$}

To probe the polarity of $\mathrm{HBsAg}$ and $\mathrm{HBx}$, the zeta potential was measured. The zeta potential of HBsAg and HBx in $10 \mathrm{mM} \mathrm{BTP} \mathrm{(in} \mathrm{pH} \mathrm{7)} \mathrm{was}-9.00 \mathrm{mV}$ and $7.653 \mathrm{mV}$, respectively. The results revealed that $\mathrm{HBs} A g$ and $\mathrm{HBx}$ proteins possessed negative and positive polarities, respectively. This result is consistent with studies that showed the isoelectric point (pI) of HBsAg [39] and HBx [40] were 4.6 and 8.3, respectively. It is known that when the $\mathrm{pH}$ value is greater than $\mathrm{pI}$, the protein surface is negatively charged or vice versa [41]. Hence, the $\mathrm{pH} 7$ buffer that has been used in this system would cause HBsAg to carry a negative charge, whereas HBx carries a positive charge.

Figure 4 shows the electrical properties of functionalized pSiNWFET response on the various concentration of HBsAg and $\mathrm{HBx}$. Figure $4 \mathrm{~A}$ shows the biosensing of $\mathrm{HBsAg}$ using an HBsAb-immobilized pSiNWFET. The electrical property of a pSiNWFET was conducted at a fixed drain voltage $\left(\mathrm{V}_{\mathrm{D}}=0.5 \mathrm{~V}\right)$ and gate voltage sweeping from $0.8 \mathrm{~V}$ to $2.0 \mathrm{~V}$. Firstly, the baseline of the pSiNWFET was measured and revealed in black line (G1). Subsequently, the concentration of $100 \mathrm{fg} / \mathrm{mL}$ of HBsAg was loaded onto the device and incubated for $30 \mathrm{~min}$. The analyte was removed and replaced with the $10 \mathrm{mM}$ Bis-tris propane on the device. The pSiNWFET showed a decrease in $\mathrm{I}_{\mathrm{D}}$ and resulted in a positive shift in the threshold voltage (red line, G2). Later, the higher concentration of HBsAg $\left(1 \mathrm{pg} / \mathrm{mL}\right.$ or $10 \mathrm{pg} / \mathrm{mL}$ ) was repeated for the above-mentioned steps. The decreased $\mathrm{I}_{\mathrm{D}}$ trend was obtained for $1 \mathrm{pg} / \mathrm{mL}$ (blue line, G3) and $10 \mathrm{pg} / \mathrm{mL}$ (green line, G4) of HBsAg compared to baseline. The normalized value of each sample group was calculated, and the average of 3 devices was presented in the inset figure. The threshold voltage (Figure S3) and the value of threshold voltage changing $\left(\Delta \mathrm{V}_{\text {th }}\right)$ were calculated. The normalized value of G2-G1 was $120.262 \mathrm{mV}$, and an increasing trend was observed for G3-G1 (330.728 mV) and G4-G1 (432.247 mV). 
(A)

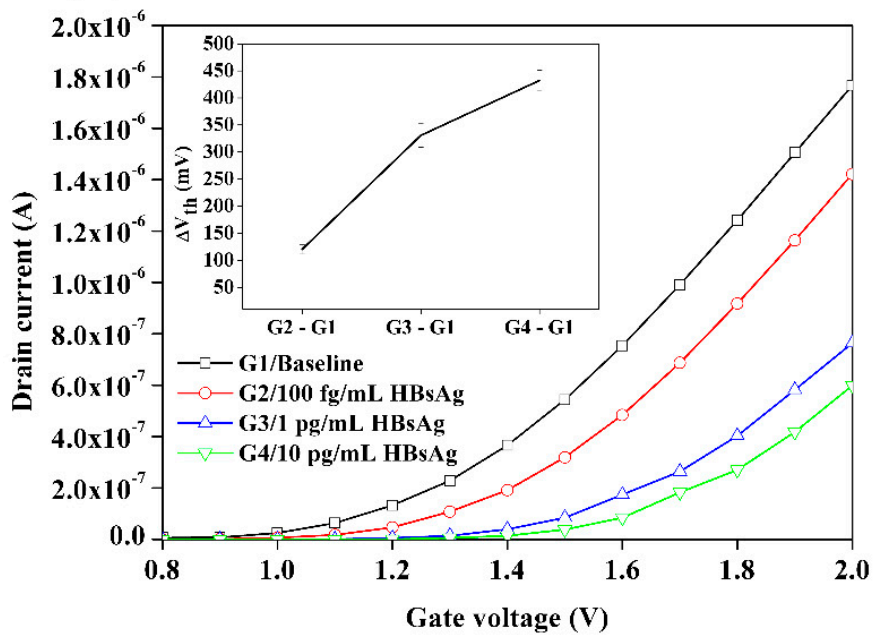

(B)

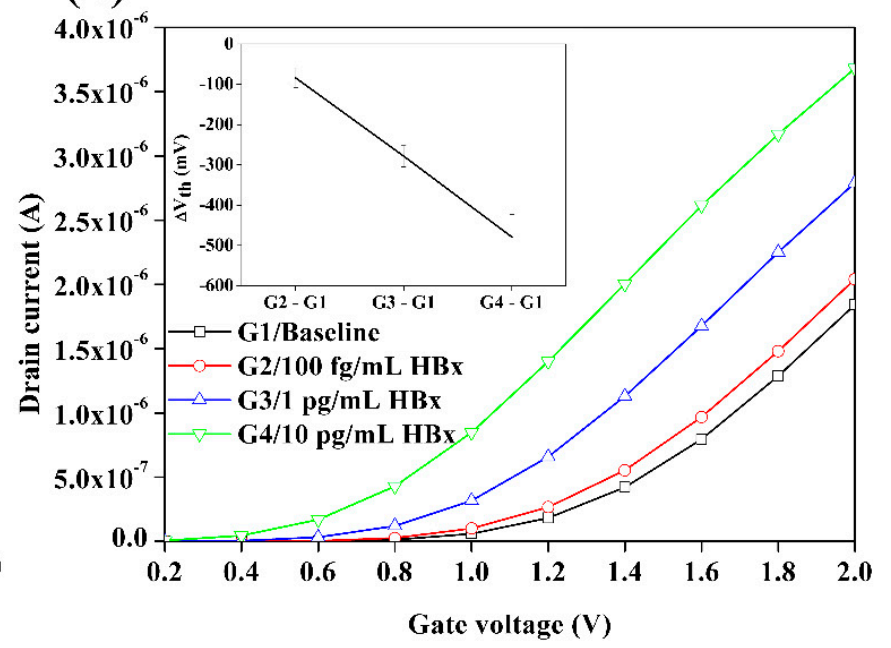

Figure 4. The electrical properties of pSiNWFET response on the various concentration of HBsAg and HBx. (A) The electrical property of the pSiNWFET was conducted at a fixed drain voltage $\left(V_{D}=0.5 \mathrm{~V}\right)$ and gate voltage sweeping from $0.8 \mathrm{~V}$ to $2.0 \mathrm{~V}$. The black line indicates baseline (G1) of the pSiNWFET; red line, blue line, and green line indicate the electrical property of pSiNWFET after being incubated with $100 \mathrm{fg} / \mathrm{mL} \mathrm{HBsAg}(\mathrm{G} 2), 1 \mathrm{pg} / \mathrm{mL} \mathrm{HbsAg}(\mathrm{G} 3)$, and $10 \mathrm{pg} / \mathrm{mL} \mathrm{HBsAg}$ (G4), respectively. The inset figure shows changes in the threshold voltage after each sample's incubation, and the error bar indicates standard error from three devices. (B) The electrical property of the pSiNWFET was conducted at a fixed drain voltage $\left(\mathrm{V}_{\mathrm{D}}=0.5 \mathrm{~V}\right)$ and gate voltage sweeping from $0.2 \mathrm{~V}$ to $2.0 \mathrm{~V}$. The black line indicates baseline (G1) of the pSiNWFET; red line, blue line, and green line indicate the electrical property of pSiNWFET after being incubated with $100 \mathrm{fg} / \mathrm{mL} \mathrm{HBx}$ (G2), $1 \mathrm{pg} / \mathrm{mL} \operatorname{HBx}(\mathrm{G} 3)$, and $10 \mathrm{pg} / \mathrm{mL} \operatorname{HBx}(\mathrm{G} 4)$, respectively. The inset figure shows changes in the threshold voltage after each sample incubation, and the error bar indicates standard error from three devices.

Similarly, Figure 4B showed the electrical property of the anti-HBx-immobilized pSiNWFET in biosensing of HBx. The test was conducted at a fixed drain voltage $\left(\mathrm{V}_{\mathrm{D}}=0.5 \mathrm{~V}\right)$, and gate voltage sweeps from $0.2 \mathrm{~V}$ to $2.0 \mathrm{~V}$. The black line indicates the baseline (G1) of the pSiNWFET, whereas the red, blue, and green lines indicated the electrical property of pSiNWFET after incubating with $100 \mathrm{fg} / \mathrm{mL}$ HBx (G2), $1 \mathrm{pg} / \mathrm{mL} \mathrm{HBx}$ (G3), and $10 \mathrm{pg} / \mathrm{mL}$ $\mathrm{HBx}$ (G4), respectively. The inset figure presented $\Delta \mathrm{V}_{\text {th }}$ of the average value of three devices after each sample incubation. The normalized value of G2-G1 was $-84.005 \mathrm{mV}$, then the value decreased to $-278.552 \mathrm{mV}$ for G3-G1 and to $-479.085 \mathrm{mV}$ for G4-G1.

Figure 4 demonstrated that the n-type pSiNWFETs have an outstanding and consistent response to negative-charge and positive-charge proteins. The increasing concentration of HBsAg resulted in a decreased $I_{D}$ trend compared to baseline shows that it is a negatively charged protein. Without ambiguity, the pSiNWFET showed a contrary response to HBx biosensing. An increased concentration of $\mathrm{HBx}$ showed an increasing $\mathrm{I}_{\mathrm{D}}$ trend indicates a positively charged protein. According to the general sensing mechanism of SiNWFET, the antigen-antibody interaction on the surface of the sensing area causes the channel conductance to change according to the charge accumulation. In other terms, for an n-type SiNWFET, when negatively charged antigen binds to the antibody immobilized on the sensor surface, it introduces an accumulation of negative charges on the pSiNWFET surface and subsequently decreases the drain current. On the contrary, the accumulation of positive charges on the pSiNWFET surface increased the magnitude of the drain current [23]. The results are consistent with the zeta potential measurement of the analyte proteins.

Furthermore, the pSiNWFET demonstrated its ultrahigh-sensitive properties in the biosensing of HBV-related proteins. Radioimmunoassay (RIA) or enzyme immunoassays (EIA) are the general serological method to determine HBV infection [6]. This study demonstrates the detection of the lowest concentration of HBsAg that can be detected using pSiNWFET was $100 \mathrm{fg} / \mathrm{mL}$. This sensitivity value is comparable to the commercialized fully automated assays invented by Abbott Diagnostic and Roche Diagnostic, which have 
the lowest detection limit at $0.2 \mathrm{ng} / \mathrm{mL}$ [6]. The higher sensitivity achieved by a SiNWFET sensor is due to the structure of silicon nanowire (SiNW). The SiNW 1-dimensional (1-D) nanomaterial has a high surface-area-to-volume ratio that improves analytical sensitivity in bio-molecules detection. This is because the analytes binding onto the surface of SiNW will cause a bulky depletion or accumulation of carrier of nanometer diameter structure and increase the sensitivity to femto-molar detection limits [34]. In addition, our pSiNWFET was able to detect HBx at the lowest concentration of $100 \mathrm{fg} / \mathrm{mL}$, which was comparable to EIA with a detection limit at the sub-nano range [42]. The sensing principle of SiNWFET allows for the direct conversion of bio-molecule events on the sensing surface to the detectable electrical signal without requiring amplification and provides a direct detection method of protein-protein interaction.

\subsection{Equilibrium Dissociation Constant $\left(K_{D}\right)$ of Protein-Protein Interaction on pSiNWFET}

In addition to the biosensing of biomolecules using pSiNWFET, the threshold voltage shift upon various concentrations of the analytes could be used to extract the equilibrium constant associated with the behavior of binding and desorption of the protein-protein interaction on the device surface. As shown in Figure 5, the red line indicates the threshold voltage increase accordingly to the increased HBsAg concentration, whereas the blue line indicates the threshold voltage decreases accordingly to the increased HBx concentration. Furthermore, the extraction of the equilibrium dissociation constant $\left(K_{D}\right)$ was obtained by applying Equation (1) in the aforementioned. Accordingly, the values of the extracted $K_{D}$ for HBsAg-HBsAb, and HBx-anti-HBx in this study were approximately $12 \mathrm{fM}$ and $40 \mathrm{fM}$, respectively (Figure 5). In the past, the powerful tool to determine $K_{D}$ was surface plasmon resonance (SPR), but the integration of SPR requires expensive optical components that have limited its application [32]. Recently, the use of label-free SiNWFET platforms to determine the equilibrium constant describing the behavior of the proteins affinity kinetics has been demonstrated [32], revealing the equilibrium constant of fM levels for the protein interactions, which is in agreement with our findings.

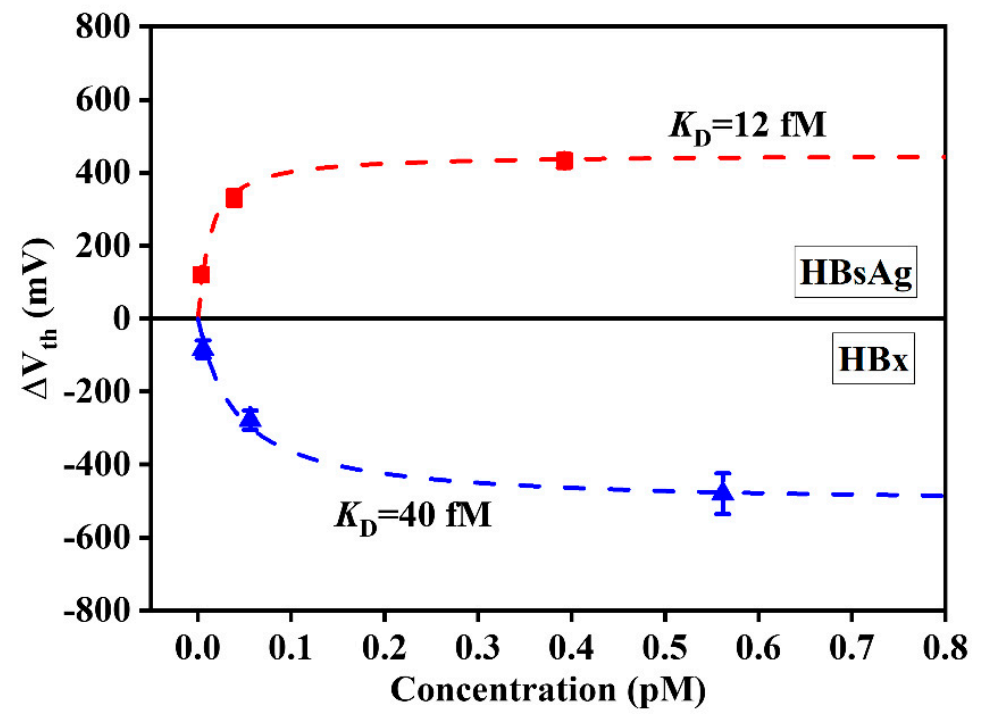

Figure 5. The threshold voltage changes and $K_{D}$ of HBsAg and HBx biosensing. The red line indicates that the threshold voltage increases accordingly to the increased HBsAg concentration, whereas the blue line indicates that the threshold voltage decreases accordingly to the increased HBx concentration. The extraction $K_{D}$ of HBsAg and $\mathrm{HBx}$ were obtained at $12 \mathrm{fM}$ and $40 \mathrm{fM}$, respectively. The error bar was indicated the standard error of three devices.

Taking the amount of the contributed electric charge for each HBsAg and HBx protein as high as one charge, the surface density of binding sites on the surface of pSiNWFET can be approximately estimated using Equation (1). The $C_{0}$ is the planar capacitance 
featuring the stacked silicon nitride (50 $\mathrm{nm}$ in thickness)/silicon oxide layer ( $85 \mathrm{~nm}$ in thickness). As a result, the maximum surface density $[B]_{\text {max }}$ was extracted to be at the level of $\sim 10^{13} / \mathrm{cm}^{2}$ for the HBsAg and HBx proteins, consistent with the typical values reported in the literature $[43,44]$.

\subsection{Prospective of pSiNWFET Sensor as Multiplexing Biochip for HBV Detection in Monitoring Patients' Status}

To date, various types of immunosensors have been proposed for HBV infection diagnosis [45], including HBV X gene detection [46], and HBsAg detection [29,30]. These sensors were developed to address the disadvantages of current diagnosis methods, such as the required bulky instruments or complicated procedures. In other words, the ultimate goal is to develop a reliable and feasible tool for simple, sustainable, and cost-effectively diagnosis of HBV infection and the long-term monitoring of $\mathrm{CHB}$ patients. A list of other FET-based sensors related to HBV detection has been presented in Table 1 . In this paper, the CMOS-compatible fabrication technique was adopted to fabricate the pSiNWFETs $[25,28]$, which was successfully demonstrated in a commercial process foundry in Taiwan. It is showing a great opportunity of commercially using the pSiNWFET for HBV-related proteins biosensing applications. Our pSiNWFET demonstrated its capability in detecting the femto-range concentration of target proteins, which is comparable to other FET-based sensors (Table 1). The lower concentration detection of HBV-related proteins or biomarkers could positively impact hepatitis diagnosis, particularly monitoring the titer of CHB-related biomarkers. In addition, the achievement of the functionalization technique and maturity of the semiconductor industry provides an opportunity to develop a multiplexing HBV biomarkers detection biochip. The biochip could be immobilized with multiple types of HBV infection diagnosis biomarkers and provide high-throughput biosensing.

Table 1. List of other FET-based sensors related to HBV detection.

\begin{tabular}{cccc}
\hline References & Type of FET & Analyte & Detection Range \\
\hline$[29]$ & $\begin{array}{c}\text { Tailored-Dual Gate FET } \\
\text { Electrochemically } \\
\text { reduced graphene oxide }\end{array}$ & HBsAg & $1.5 \mathrm{fM}-1.5 \mathrm{nM}$ \\
{$[30]$} & FET & HBsAg & $1 \mathrm{fM}-20 \mathrm{pM}$ \\
& NWFET & HBx (DNA) & $10 \mathrm{fM}-1 \mathrm{pM}$ \\
{$[46]$} & pSiNWFET & HBsAg & $3.92 \mathrm{fM}-0.39 \mathrm{pM} ;$ \\
This paper & HBx (protein) & $5.61 \mathrm{fM}-0.56 \mathrm{pM}$ \\
\hline
\end{tabular}

As aforementioned in Section 3.4, the detection mechanism of SiNWFET response to the analytes was based on the sensor surface depletion or accumulation of the charge. In contrast to nucleotide detection that detects a negative carrier using NWFET, the uncertainty of the analyte protein net charge towards a $\mathrm{pH}$ range would affect the interpretation of the sensor response in the protein detection [47]. In this paper, the pSiNWFET demonstrated can differentiate two opposite net charges of the proteins in a given $\mathrm{pH} 7$ by evaluating the electrical properties response. These results revealed a possibility of using pSiNWFET in determining the unknown net charge of a protein via evaluating the electrical properties.

Although early diagnosis or monitoring of hepatitis infection could be achieved by simple, ultrahigh-sensitive, and affordable pSiNWFET. The pSiNWFET also faced a few challenges, including the robustness of the devices towards detecting the real sample. Real samples, such as serum or whole blood, would cause inconsistent results due to non-specific proteins adsorption onto the sensing area and eventually causing noise to the sensor. This could be overcome by optimizing the blocking step of surface modification or by applying anti-fouling pre-treatment on the sensor. Besides that, the washing step commonly used in EIA could be adopted in the biosensing procedure to remove non-covalent binding on the 
non-specific protein adsorption. However, the efficiency of adopting the washing step has yet to be identified.

\section{Conclusions}

In this paper, we have used the pSiNWFET biosensor fabricated by the commercial process foundry for specific, ultrahigh-sensitive, and label-free detection of HBV-related proteins, namely HBsAg and HBx. In addition to measuring the electrical properties of the pSiNWFETs to confirm the stability and reliability, the validity of the chemical functionalization process of the pSiNWFETs was confirmed using XPS and SEM. Furthermore, the functionalized pSiNWFETs were used to detect two biomarkers, HBsAg and HBx, that carried negative and positive charges, respectively, which were determined by the zeta potential measurement. The experimental results of the threshold voltage shift in pSiNWFETs consistently reflect the charge polarity of the protein targets. In addition, we suggested that pSiNWFET could be (1) a potential candidate for multiplexing detection of HBV-related biomarkers; (2) the electrical noise could be reduced by optimizing the blocking step of functionalizing or adapting the washing step. We expect this approach to positively impact hepatitis B infection diagnosis and widen the study of $\mathrm{HBsAg}$ and $\mathrm{HBx}$ concentration in HCC development.

Supplementary Materials: The following are available online at https:/ /www.mdpi.com/article/ 10.3390/bios11110442/s1, Figure S1: XPS oxygen spectra of four silicon wafer surface modifications, Figure S2: The electrical properties of three baselines of one functionalized device and nine nude devices, Figure S3: The extraction of threshold voltage of pSiNWFET, Figure S4: The top-view SEM image of the device, Figure S5: The SEM images of the HBsAb immobilization process.

Author Contributions: Conceptualization, S.K.Y. and Y.-S.Y.; methodology, S.K.Y. and S.-K.S.; validation, S.K.Y., S.-K.S., C.-W.C., Y.-Y.W. and Y.-S.Y.; data curation, S.K.Y.; writing, original draft preparation, S.K.Y.; writing, review and editing, S.K.Y., M.-P.L. and Y.-S.Y.; project administrationM.-P.L. and Y.-S.Y.; funding acquisition, S.K.Y., M.-P.L. and Y.-S.Y. All authors have read and agreed to the published version of the manuscript.

Funding: This study was supported financially by the Ministry of Science and Technology, Taiwan (Grant number 110-2221-E-492 -003; 109-2221-E-009-009-MY2; 109-2327-B-010-005).

Institutional Review Board Statement: Not applicable.

Acknowledgments: We thank Episil Holding Inc., Taiwan, for device fabrication.

Conflicts of Interest: The authors declare no conflict of interests.

\section{References}

1. Iannacone, M.; Guidotti, L.G. Immunobiology and pathogenesis of hepatitis B virus infection. Nat. Rev. Immunol. 2021, 1-14. [CrossRef]

2. Yuen, M.F.; Chen, D.S.; Dusheiko, G.M.; Janssen, H.L.A.; Lau, D.T.Y.; Locarnini, S.A.; Peters, M.G.; Lai, C.L. Hepatitis B virus infection. Nat. Rev. Dis. Primers 2018, 4, 1-20. [CrossRef] [PubMed]

3. World Health Organization. Global Hepatitis Report 2017. World Health Organization. 2017. Available online: https://apps.who. int/iris/handle/10665/255016 (accessed on 9 October 2021).

4. World Health Organization. Global Health Sector Strategy on Viral Hepatitis 2016-2021. Towards Ending Viral Hepatitis. World Health Organization. 2016. Available online: https://apps.who.int/iris/handle/10665/246177 (accessed on 9 October 2021).

5. Chan, H.L.; Thompson, A.; Martinot-Peignoux, M.; Piratvisuth, T.; Cornberg, M.; Brunetto, M.R.; Tillmann, H.L.; Kao, J.H.; Jia, J.D.; Wedemeyer, H.; et al. Hepatitis B surface antigen quantification: Why and how to use it in 2011-A core group report. J. Hepatol. 2011, 55, 1121-1131. [CrossRef] [PubMed]

6. Kim, S.-H. ELISA for Quantitative Determination of Hepatitis B Virus Surface Antigen. Immune Netw. 2017, 17, 451-459. [CrossRef] [PubMed]

7. Höner Zu Siederdissen, C.; Cornberg, M. The role of HBsAg levels in the current management of chronic HBV infection. Ann. Gastroenterol. 2014, 27, 105-112. [PubMed]

8. Tang, K.F.; Xie, J.; Chen, M.; Liu, Q.; Zhou, X.Y.; Zeng, W.; Huang, A.L.; Zuo, G.Q.; Wang, Y.; Xiang, R.; et al. Knockdown of damage-specific DNA binding protein 1 (DDB1) enhances the HBx-siRNA-mediated inhibition of HBV replication. Biologicals 2008, 36, 177-183. [CrossRef] [PubMed] 
9. Yen, C.-J.; Yang, S.-T.; Chen, R.-Y.; Huang, W.; Chayama, K.; Lee, M.-H.; Yang, S.-J.; Lai, H.-S.; Yen, H.-Y.; Hsiao, Y.-W.; et al. Hepatitis B virus $\mathrm{X}$ protein ( $\mathrm{HBx}$ ) enhances centrosomal P4.1-associated protein (CPAP) expression to promote hepatocarcinogenesis. J. Biomed. Sci. 2019, 26, 44.

10. Yue, D.; Zhang, Y.; Cheng, L.; Ma, J.; Xi, Y.; Yang, L.; Su, C.; Shao, B.; Huang, A.; Xiang, R.; et al. Hepatitis B virus X protein (HBx)-induced abnormalities of nucleic acid metabolism revealed by 1H-NMR-based metabonomics. Sci. Rep. 2016, 6, 24430.

11. Salerno, D.; Chiodo, L.; Alfano, V.; Floriot, O.; Cottone, G.; Paturel, A.; Pallocca, M.; Plissonnier, M.L.; Jeddari, S.; Belloni, L.; et al. Hepatitis B protein HBx binds the DLEU2 lncRNA to sustain cccDNA and host cancer-related gene transcription. Gut 2020, 69, 2016-2024. [CrossRef] [PubMed]

12. Zampino, R.; Boemio, A.; Sagnelli, C.; Alessio, L.; Adinolfi, L.E.; Sagnelli, E.; Coppola, N. Hepatitis B virus burden in developing countries. World J. Gastroenterol. 2015, 21, 11941-11953. [CrossRef]

13. Abbas, Z.; Siddiqui, A.R. Management of hepatitis B in developing countries. World J. Hepatol. 2011, 3, 292-299. [CrossRef] [PubMed]

14. Wait, S.; Chen, D.S. Towards the eradication of hepatitis B in Taiwan. Kaohsiung J. Med. Sci. 2012, 28, 1-9. [CrossRef]

15. Kaisti, M. Detection principles of biological and chemical FET sensors. Biosens. Bioelectron. 2017, 98, 437-448. [CrossRef] [PubMed]

16. Chen, K.-I.; Li, B.-R.; Chen, Y.-T. Silicon nanowire field-effect transistor-based biosensors for biomedical diagnosis and cellular recording investigation. Nano Today 2011, 6, 131-154. [CrossRef]

17. Lin, C.H.; Hung, C.H.; Hsiao, C.Y.; Lin, H.C.; Ko, F.H.; Yang, Y.S. Poly-silicon nanowire field-effect transistor for ultrasensitive and label-free detection of pathogenic avian influenza DNA. Biosens. Bioelectron. 2009, 24, 3019-3024. [CrossRef] [PubMed]

18. Huang, Y.-W.; Wu, C.-S.; Chuang, C.-K.; Pang, S.-T.; Pan, T.-M.; Yang, Y.-S.; Ko, F.-H. Real-Time and Label-Free Detection of the Prostate-Specific Antigen in Human Serum by a Polycrystalline Silicon Nanowire Field-Effect Transistor Biosensor. Anal. Chem. 2013, 85, 7912-7918. [CrossRef]

19. Su, P.C.; Chen, B.H.; Lee, Y.C.; Yang, Y.S. Silicon Nanowire Field-Effect Transistor as Biosensing Platforms for Post-Translational Modification. Biosensors 2020, 10, 213. [CrossRef]

20. Zafar, S.; D’Emic, C.; Jagtiani, A.; Kratschmer, E.; Miao, X.; Zhu, Y.; Mo, R.; Sosa, N.; Hamann, H.; Shahidi, G.; et al. Silicon Nanowire Field Effect Transistor Sensors with Minimal Sensor-to-Sensor Variations and Enhanced Sensing Characteristics. ACS Nano 2018, 12, 6577-6587. [CrossRef]

21. Shen, M.-Y.; Li, B.-R.; Li, Y.-K. Silicon nanowire field-effect-transistor based biosensors: From sensitive to ultra-sensitive. Biosens. Bioelectron. 2014, 60, 101-111. [CrossRef]

22. Sheikh, N.J.; Sheikh, O. Forecasting of biosensor technologies for emerging point of care and medical IoT applications using bibliometrics and patent analysis. In Proceedings of the 2016 Portland International Conference on Management of Engineering and Technology (PICMET), Honolulu, HI, USA, 4-8 September 2016. [CrossRef]

23. Syu, Y.-C.; Hsu, W.-E.; Lin, C.-T. Review-Field-Effect Transistor Biosensing: Devices and Clinical Applications. ECS J. Solid State Sci. Technol. 2018, 7, Q3196-Q3207. [CrossRef]

24. Pateraki, M.; Fysarakis, K.; Sakkalis, V.; Spanoudakis, G.; Varlamis, I.; Maniadakis, M.; Lourakis, M.; Ioannidis, S.; Cummins, N.; Schuller, B.; et al. Biosensors and Internet of Things in smart healthcare applications: Challenges and opportunities. In Wearable and Implantable Medical Devices; Academic Press: Cambridge, MA, USA, 2020; pp. 25-53.

25. Lin, H.-C.; Lee, M.-H.; Su, C.-J.; Huang, T.-Y.; Lee, C.C.; Yang, Y.-S. A simple and low-cost method to fabricate TFTs with poly-Si nanowire channel. IEEE Electron Device Lett. 2005, 26, 643-645. [CrossRef]

26. Lu, M.P.; Hsiao, C.Y.; Lai, W.T.; Yang, Y.S. Probing the sensitivity of nanowire-based biosensors using liquid-gating. Nanotechnology 2010, 21, 425505. [CrossRef] [PubMed]

27. Lin, C.H.; Feng, M.H.; Hwang, C.H.; Wu, J.Y.S.; Su, P.C.; Lin, M.Y.; Chen, C.H.; Chen, B.H.; Huang, B.Y.; Lu, M.P.; et al. Surface composition and interactions of mobile charges with immobilized molecules on polycrystalline silicon nanowires. Sens. Actuators B Chem. 2015, 211, 7-16. [CrossRef]

28. Hsiao, C.Y.; Lin, C.H.; Hung, C.H.; Su, C.J.; Lo, Y.R.; Lee, C.C.; Lin, H.C.; Ko, F.H.; Huang, T.Y.; Yang, Y.S. Novel poly-silicon nanowire field effect transistor for biosensing application. Biosens. Bioelectron. 2009, 24, 1223-1229. [CrossRef] [PubMed]

29. Lee, I.-K.; Jeun, M.; Jang, H.-J.; Cho, W.-J.; Lee, K.H. A self-amplified transistor immunosensor under dual gate operation: Highly sensitive detection of hepatitis B surface antigen. Nanoscale 2015, 7, 16789-16797. [CrossRef]

30. Basu, J.; Baral, A.; Samanta, N.; Mukherjee, N.; Roychaudhuri, C. Low Noise Field Effect Biosensor with Electrochemically Reduced Graphene Oxide. J. Electrochem. Soc. 2018, 165, B3201-B3207. [CrossRef]

31. Ortiz-Conde, A.; Sánchez, F.G.; Liou, J.J.; Cerdeira, A.; Estrada, M.; Yue, Y. A review of recent MOSFET threshold voltage extraction methods. Microelectron. Reliab. 2002, 42, 583-596. [CrossRef]

32. Duan, X.; Li, Y.; Rajan, N.K.; Routenberg, D.A.; Modis, Y.; Reed, M.A. Quantification of the affinities and kinetics of protein interactions using silicon nanowire biosensors. Nat. Nanotech. 2012, 7, 401-407. [CrossRef]

33. Hobbs, R.G.; Petkov, N.; Holmes, J.D. Semiconductor Nanowire Fabrication by Bottom-Up and Top-Down Paradigms. Chem. Mater. 2012, 24, 1975-1991. [CrossRef]

34. Noor, M.O.; Krull, U.J. Silicon nanowires as field-effect transducers for biosensor development: A review. Anal. Chim. Acta 2014, 825, 1-25. [CrossRef]

35. Wu, J.Y.; Lin, C.H.; Feng, M.H.; Chen, C.H.; Su, P.C.; Yang, P.W.; Zheng, J.M.; Fu, C.W.; Yang, Y.S. Preparation of Silicon Nanowire Field-effect Transistor for Chemical and Biosensing Applications. J. Vis. Exp. 2016, 110, 53660. [CrossRef] [PubMed] 
36. McArthur, S.L.; Mishra, G.; Easton, C.D. Applications of XPS in Biology and Biointerface Analysis. In Surface Analysis and Techniques in Biology; Smentkowski, V., Ed.; Springer International: Berlin, Germany, 2014.

37. Yan, X.; Xu, T.; Chen, G.; Yang, S.; Liu, H.; Xue, Q. Preparation and characterization of electrochemically deposited carbon nitride films on silicon substrate. J. Phys. D Appl. Phys. 2004, 37, 907-913. [CrossRef]

38. Miranda, A.; Martínez, L.; De Beule, P.A.A. Facile synthesis of an aminopropylsilane layer on $\mathrm{Si} / \mathrm{SiO}_{2}$ substrates using ethanol as APTES solvent. MethodsX 2020, 7, 100931. [CrossRef]

39. Lee, Y.S.; Kim, B.K.; Choi, E.C. Physicochemical properties of recombinant hepatitis B surface antigen expressed in mammalian cell (C127). Arch. Pharm. Res. 1998, 21, 521-526. [CrossRef] [PubMed]

40. Jameel, S.; Siddiqui, A.; Maguire, H.F.; Rao, K.V. Hepatitis B virus X protein produced in Escherichia coli is biologically functional. J. Virol. 1990, 64, 3963-3966. [CrossRef]

41. Cleaves, H.J. Isoelectric Point. In Encyclopedia of Astrobiology; Gargaud, M., Amils, R., Cernichicaro Quintanilla, J., Cleaves, H.J., Irvine, W.M., Pinti, D., Viso, M., Eds.; Springer: Berlin/Heidelberg, Germany, 2011; p. 858.

42. Zhang, S.; Garcia-D'Angeli, A.; Brennand, J.P.; Huo, Q. Predicting detection limits of enzyme-linked immunosorbent assay (ELISA) and bioanalytical techniques in general. Analyst 2014, 139, 439-445. [CrossRef]

43. Fritz, J.; Cooper, E.B.; Gaudet, S.; Sorger, P.K.; Manalis, S.R. Electronic detection of DNA by its intrinsic molecular charge. Proc. Natl. Acad. Sci. USA 2002, 99, 14142-14146. [CrossRef] [PubMed]

44. Tulzer, G.; Heitzinger, C. Fluctuations due to association and dissociation processes at nanowire-biosensor surfaces and their optimal design. Nanotechnology 2015, 26, 025502. [CrossRef]

45. Tyas, A.A.; Raeni, S.F.; Sakti, S.P.; Sabarudin, A. Recent Advances of Hepatitis B Detection towards Paper-Based Analytical Devices. Sci. World J. 2021, 2021, 6643573. [CrossRef]

46. Wu, C.-Y.; Cheng, H.-Y.; Ou, K.-L.; Wu, C.-C. Real-time sensing of hepatitis B virus X gene using an ultrasensitive nanowire field effect transistor. J. Polym. Eng. 2014, 34, 273-277. [CrossRef]

47. Poghossian, A.; Schöning, M.J. Label-Free Sensing of Biomolecules with Field-Effect Devices for Clinical Applications. Electroanalysis 2014, 26, 1197-1213. [CrossRef] 